\title{
Effects of Physical Exercise on Adiponectin, Leptin, and Inflammatory Markers in Childhood Obesity: Systematic Review and Meta-Analysis
}

\author{
Felice Sirico, MD,' Antonio Bianco, MD,' Giovanni D'Alicandro, MD, \\ Clotilde Castaldo, MD,' Stefania Montagnani, MD, Rocco Spera, MD, \\ Franca Di Meglio, MD, PhD,,* and Daria Nurzynska, MD, PhD ${ }^{1, *}$
}

\section{Abstract}

Background: New findings on adipose tissue physiology and obesity-associated inflammation status suggest that modification of the adipokine level can be relevant for the long-term prevention of obesity-associated chronic disease.

Objectives: The scope of the present study was to investigate the effectiveness of physical exercise in reducing the systemic inflammation related to obesity in children.

Methods: We conducted a systematic review with meta-analysis of controlled randomized trials, identified through electronic database search, which investigated the effect of physical exercise, without concomitant dietary intervention, on adiponectin, leptin, and/ or other inflammatory markers in children up to age 18 years with a body mass index greater than the 95th percentile for age and sex.

Results: Seven trials were included in the meta-analysis, with a total of 250 participants. Compared with the control group without any lifestyle modification, the physical exercise resulted in a reduction in leptin [standardized mean difference (SMD) $-1.13 ; 95 \%$ confidence interval $(95 \% \mathrm{CI}):-1.89$ to $-0.37 ; I^{2}=79.9 \%$ ] and interleukin-6 (SMD $-0.84 ; 95 \% \mathrm{CI}$ : -1.45 to $\left.-0.23, I^{2}=0.9 \%\right)$ and an increase in adiponectin plasma concentration (SMD 0.69; 95\%CI: $0.02-1.35 ; I^{2}=74.3 \%$ ).

Conclusions: These results indicate that physical exercise improved the inflammatory state in children with obesity. It is unclear whether this effect can reduce the risk of cardiovascular and metabolic disease in adulthood. Clinical trials with a uniform intervention protocol and outcome measurements are required to put our knowledge on adipose tissue biology into a clinical perspective.

Keywords: adiponectin; childhood obesity; inflammation; leptin; physical exercise

\section{Introduction}

N otwithstanding the evidence linking excess body fat at a young age to type 2 diabetes and coronary heart disease in adult years, ${ }^{1}$ childhood obesity continues to rise in Europe. ${ }^{2}$ Based on experimental data accumulated over the last decade, adipose tissue is a metabolically active organ, able to respond to incoming signals and secrete several adipokines involved in modulating the metabolic processes and inflammatory responses in an endocrine manner. ${ }^{3}$ Circulating levels of adipokines reach a characteristic profile in specific metabolic states. ${ }^{4} \mathrm{Im}$ portantly, logistic analysis indicates that some peptide hormones released by adipocytes are strictly associated with obesity. ${ }^{5}$ Leptin and adiponectin are two such adipokines that act on several target organs, including the brain, liver, pancreas, muscle, immune system, and adipose tissue itself.

\footnotetext{
'Department of Public Health, Human Anatomy, and Sports Medicine Section, University of Naples "Federico II," Naples, Italy.

${ }^{2}$ Department of Neuroscience and Rehabilitation, Centre for Sports Medicine and Disability, Santobono-Pausilipon Children's Hospital, Naples, Italy.

*These authors are joint senior authors.

(c) Felice Sirico et al., 2018; Published by Mary Ann Liebert, Inc. This Open Access article is distributed under the terms of the Creative Commons Attribution Noncommercial License (http://creativecommons.org/licenses/by-nc/4.0/) which permits any noncommercial use, distribution, and reproduction in any medium, provided the original author(s) and the source are credited.
} 
Leptin is able to signal energy sufficiency; its action on the central nervous system, mainly hypothalamic neuroendocrine cells, and on peripheral tissues, including muscle and pancreatic islet cells, results in reduced food intake and increased energy expenditure. ${ }^{3}$ Interestingly, common forms of obesity are characterized by an elevated, rather than reduced, concentration of leptin in plasma, ${ }^{6}$ which suggests leptin resistance in individuals with obesity. ${ }^{7}$ The main targets of adiponectin are the liver and skeletal muscle, where adiponectin acts to reduce glucose production and to increase insulin sensitivity and energy expenditure. In contrast to leptin, adiponectin levels are usually reduced in subjects with obesity. ${ }^{5,8}$

Apart from their metabolic effects, both adipokines are involved in inflammation and immune response, with leptin having proinflammatory and adiponectin having antiinflammatory properties. In particular, high levels of leptin activate monocytes and macrophages to produce IL-6 and tumor necrosis factor (TNF) $\alpha$. The latter stimulates expression of leptin and its receptor. One effect of adiponectin is the reduction of TNF $\alpha$ production in macrophages. ${ }^{9}$ On the one hand, the levels of leptin (positively) and adiponectin (negatively) correlate with body mass in$\operatorname{dex}(\mathrm{BMI})^{10}$; on the other hand, obesity is associated with a low-grade systemic inflammation, ${ }^{11}$ which has been correlated with cardiovascular events, metabolic syndrome, and diabetes. ${ }^{12}$ In light of these relationships, a continuum emerges between obesity, inflammation, and metabolic disease. Given the role of adipokines in inflammation and cardiovascular pathophysiology, development of effective and validated methods to control obesity in childhood is a rational approach to prevent major diseases in adulthood. ${ }^{13}$

Childhood obesity does not permanently increase cardiovascular risk provided that it is successfully treated. However, bad habits are difficult to eradicate and, according to studies, over $80 \%$ of those with obesity as children have obesity as adults. ${ }^{1}$ In children, making better food choices and becoming active are the most commonly suggested measures; the same generally applies to adults with overweight and obesity. However, the loss of body weight cannot be the main target of these lifestyle interventions. First, contrary to the loss of body fat, the loss of lean mass can have negative effects on mortality; second, loss of body fat does not offer the same benefits as an increase in lean mass. ${ }^{14}$ From these observations, it follows that the type and modality of intervention can be essential to the overall goal; the interventions that target systemic inflammation could help to reduce chronic disease risk in the long term.

Systematic reviews of interventions aimed at reducing obesity have considered a combined effect of exercise and diet on body mass, body composition, lipid, and glucose levels. ${ }^{15,16}$ Diet and exercise, however, can have different effects on adipose tissue distribution, composition, and activity. It has been shown that being physically active reduces the risk of all-cause mortality ${ }^{17}$ and that positive effects of physical exercise extend well beyond its effect on BMI. ${ }^{18}$ In the light of new findings on adipose tissue physiology and obesity-associated inflammation status, modification of the adipokine level and signaling due to changes in adipose tissue quality, rather than quantity, can be relevant for the long-term prevention of obesity-associated chronic disease. Hence, we have chosen to summarize, through a systematic approach, available evidence on the impact of physical activity on adiponectin, leptin, and other inflammatory markers in children with obesity.

\section{Methods}

The study followed the Preferred Reporting Items for Systematic Reviews and Meta-Analyses (PRISMA) Guidelines. ${ }^{19}$ The PRISMA Checklist is reported in the Supplementary Table S1; (Supplementary Data are available online at www.liebertpub.com/chi).

\section{Search Strategy}

The following databases were searched from inception to March 2017: PubMed, Web of Science, Medline, Scopus, and CINAHL databases. English language restriction was applied. According to the syntax rules of each database, the following terms were logically combined to search sources related to the main topic of the analysis: obesity AND (children OR childhood) AND [(inflammation OR inflammatory markers OR adiponectin OR leptin OR (interleukin6 OR IL-6) OR (CRP OR C-reactive protein) OR (TNF- $\alpha$ OR tumor necrosis factor- $\alpha$ )] AND (physical exercise OR physical activity OR sport OR exercise). Full search strategy for PubMed database is reported in the Supplementary Table S2. Observational studies, cohort studies, narrative and systematic reviews, case reports, and case series were excluded. In addition, the reference lists of relevant articles were scanned to identify other published and not indexed sources. All studies identified through database and manual searches were collected in a reference manager software. After removal of duplicates, two authors (F.S. and A.B.) independently reviewed the titles and abstracts. Inconsistencies were discussed with another author (D.N.). The two authors who reviewed the titles and abstracts then independently analyzed the full text of the remaining articles to determine final inclusion.

\section{Selection Criteria}

The inclusion criteria were as follows: (1) participants male and/or female at a mean age of 18 years or younger, with obesity, defined as BMI greater than the 95th percentile for age and sex, (2) supervised intervention consisting in physical exercise programme, (3) comparison to control with no change in lifestyle or activity, (4) outcome consisting in a change of the inflammatory marker level, and (5) controlled randomized and nonrandomized trials published in English language. Studies with combined interventions of any type and, in particular, those with concomitant nutritional counseling or dietary intervention were excluded. Also, data regarding normal weight or overweight patients were not considered. 


\section{Data Extraction}

Two authors (F.S. and A.B.) independently extracted data from selected studies, compiling a purposefully designed form, including the following: study characteristics (first author's surname, year of publication, journal of publication), participant characteristics (sample size, age, and sex), experimental design (groups of treatment, type, frequency, and intensity of physical activity administered as the intervention, length of intervention in weeks), outcome assessment (main outcome measures and type of inflammatory marker measured, changes in the outcome). Another author (D.N.) compared the extraction forms; all differences were reviewed, discussed and corrected. The corresponding authors of the studies that met all inclusion criteria, but did not report sufficient quantitative data, were contacted by email. If data were not received, the study was considered for qualitative analysis only.

\section{Risk of Bias}

Studies included in qualitative and quantitative analysis were assessed independently for risk of bias by two authors (F.S. and A.B.), using the guidelines included in the Cochrane Handbook for Systematic Review of Interventions. ${ }^{20}$ The following sources of bias were assessed: random sequence generation, allocation concealment, blinding of participants and personnel, blinding of outcome assessment, incomplete outcome data, selective reporting, and other sources of bias (specified and explained in each case). Each domain was given a quality score of high or low risk of bias. If data were insufficient to make a reasonable judgment, the domain was classified as unclear risk of bias. Another author (D.N.) was consulted when discrepancies occurred on comparison of the two independent assessments. We did not perform the Egger test to assess the publication bias because the number of included studies was lower than 10 . The risk of bias was reported graphically using Review Manager v.5.3 software. ${ }^{21}$

\section{Statistical Analyses}

All analyses were performed using STATA software v.12 (StataCorp LP). The effect size and 95\% confidence intervals $(95 \% \mathrm{CI})$ of each included study were calculated using the metan routine. The number of patients, mean difference, and pooled standard deviation were considered for each group of treatment. When quantitative data regarding outcome were reported in different units, the effect size was calculated as standardized mean difference (SMD). The mean difference and standard deviation of parameters from preintervention and postintervention were calculated, as suggested by the Cochrane guidelines for the analysis of clinical trials. For those studies that randomized participants with obesity into three groups (e.g., control group and two intervention groups with different exercise protocols), the data of every intervention group were independently compared with the data of the control group.

The random-effects model was chosen and the presence of heterogeneity was assessed using the Q statistics, based on the DerSimonian-Laird approach. ${ }^{22}$ The heterogeneity variance $\tau^{2}$ was measured. $I^{2}$ was used to express heterogeneity as percentage. A $Q$ value with a significance of $p$ less than or equal to 0.05 (two-tailed) was considered significant to reject the null hypothesis. All $p$-values greater than 0.05 were evaluated according to the statistical power of the test. Significant heterogeneity was considered present when $p<0.05$ and $I^{2}>60 \%$. The impact of each study on the summary effect was evaluated using the METAN INF command.

\section{Results}

\section{Study Selection and Characteristics}

Results of the systematic search and selection of studies are reported as a PRISMA recommended flow diagram in Figure 1. The literature search, after removal of duplicates, identified 1881 articles containing the indicated keywords. Thirty-eight studies had their full text analyzed for inclusion criteria. As a result of this selection, seven studies were included in the qualitative synthesis. Four studies examined changes in adiponectin, ${ }^{23-26}$ four examined changes in leptin, ${ }^{25-28}$ and three evaluated at least one other inflammatory marker (IL-6, CRP, TNF- $\alpha$, plasminogen activator inhibitor-1, or resistin) preintervention and postintervention. ${ }^{24,22,29}$ One study, however, was excluded from quantitative analysis because it did not report numerical data regarding the evaluation of the outcome. ${ }^{29}$

Characteristics of the included studies are summarized in Table 1. Duration of intervention, as well as type, frequency, and intensity of exercise differed between studies. According to the authors of the included studies, all the observed effects were considered in relationship to the supervised exercise only. In three studies, ${ }^{25,26,29}$ the authors specified that all participants were instructed not to change their regular physical activity habits or diet during the experiment. Only the study by Kim et al. ${ }^{24}$ did not include such statement, while in the studies by Fazelifar et al., ${ }^{23,27}$ all participants were actually requested to avoid doing any exercise other than that required by the study protocol. Karacabey ${ }^{28}$ mentioned that all the study participants undertook the same diet program without providing any further details.

\section{Risk of Bias}

Owing to the type of intervention, the blinding of participants was not feasible, hence, it was not considered relevant in the assessment of the bias risk. Similarly, a low rating was attributed to blinding of the outcome assessment because the main outcome was represented by a numeric value obtained from independent laboratory tests. The authors had no conflicts of interest to declare in relationship to their studies. Consequently, selection, attrition, reporting, and other possible biases were the main indicators of the validity of the included studies. Risk of bias assessment is reported graphically in Figure 2. The funnel plots of standard error of SMD against SMD for 


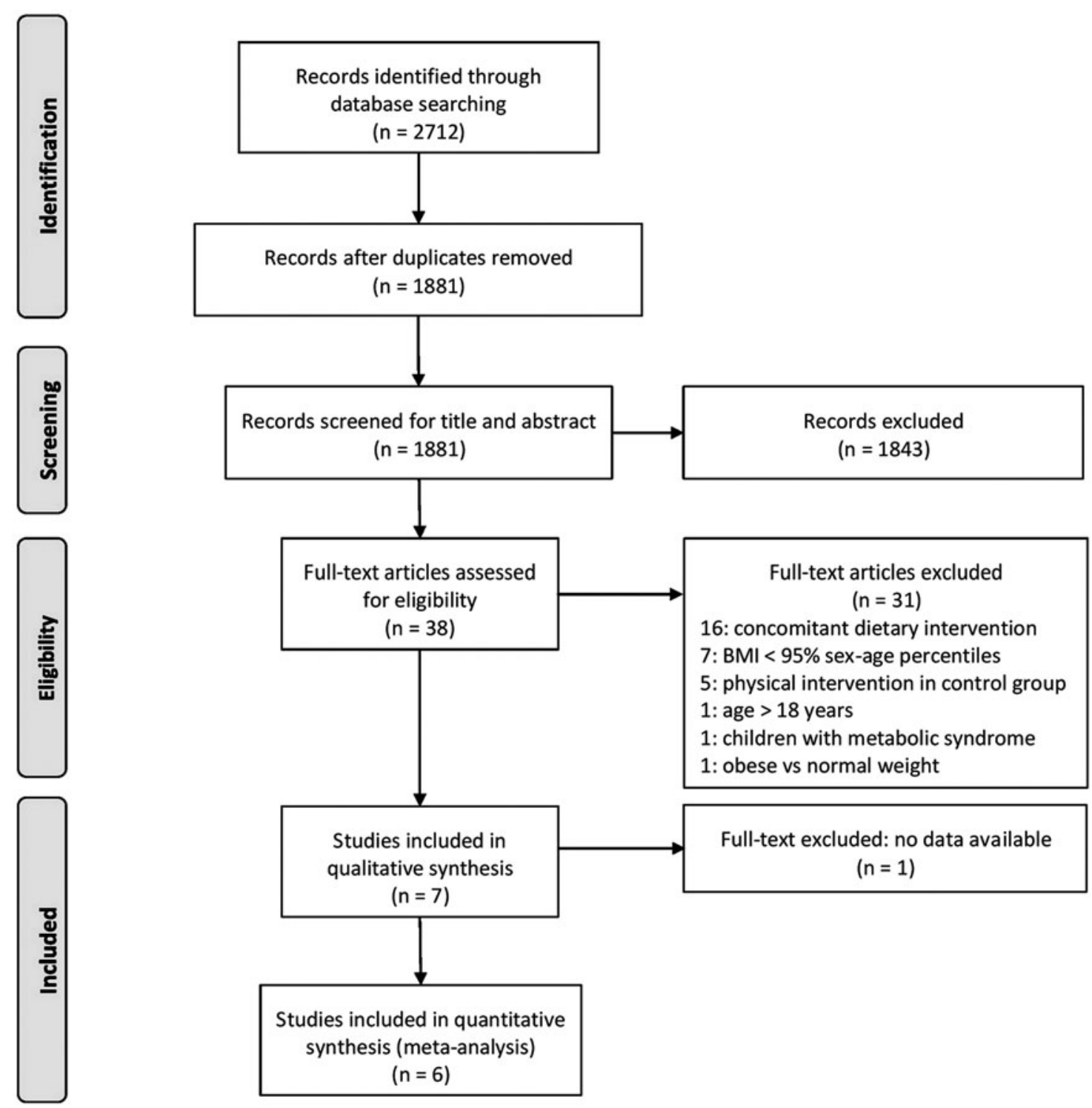

Figure 1. Preferred reporting items for systematic reviews and meta-analyses flow diagram of the study selection process. BMI, body mass index.

adiponectin and leptin studies were symmetric and did not suggest publication bias (Supplementary Fig. S1).

Two studies by Fazelifar et al. ${ }^{23,27}$ raised concerns about the possibility of additional bias because they had numerous inconsistencies in the reporting and interpreting of findings, respectively, in the results and discussion sections. Moreover, it is possible that both concurrent publications actually reported the split results of the study on the same sample without proper cross-referencing. For these reasons those redundant publications were considered at a high risk of bias. In the study by Monteiro et al., ${ }^{29}$ the sequence generation was not randomized, hence, we identified it as having a high risk of selection bias. Moreover, that study failed to report numerical data regarding the levels of inflammatory cytokines, which is a reason we considered it at high risk of reporting bias.

\section{Meta-Analysis}

Both adiponectin and leptin concentrations showed significant differences in pooled effect estimates in favor of the intervention group. In particular, leptin concentration (Fig. 3) significantly decreased (SMD -1.13 [95\%CI: -1.89 to -0.37 , comparison: 0.004 ; heterogeneity $\chi^{2}=19.9$, $\left.p=0.001, I^{2}=79.9 \%, \tau^{2}=0.601\right)$, while adiponectin levels (Fig. 4) increased with respect to the control group (SMD 0.69 [95\%CI: $0.02-1.35$ ], comparison: 0.04 ; heterogeneity $\left.\chi^{2}=15.56, p=0.004, I^{2}=74.3 \%, \tau^{2}=0.427\right)$.

Other inflammatory markers evaluated in at least two studies included IL-6, CRP, and TNF- $\alpha$ (Fig. 5). Pooled data indicated that there was a significant reduction in IL-6 concentration (SMD -0.84 [95\%CI: -1.45 to -0.23 ], comparison: 0.007 ; heterogeneity $\chi^{2}=1.01, p=0.315, I^{2}=0.9 \%$, $\left.\tau^{2}=0.002\right)$ and a trend in favor of CRP reduction in the 


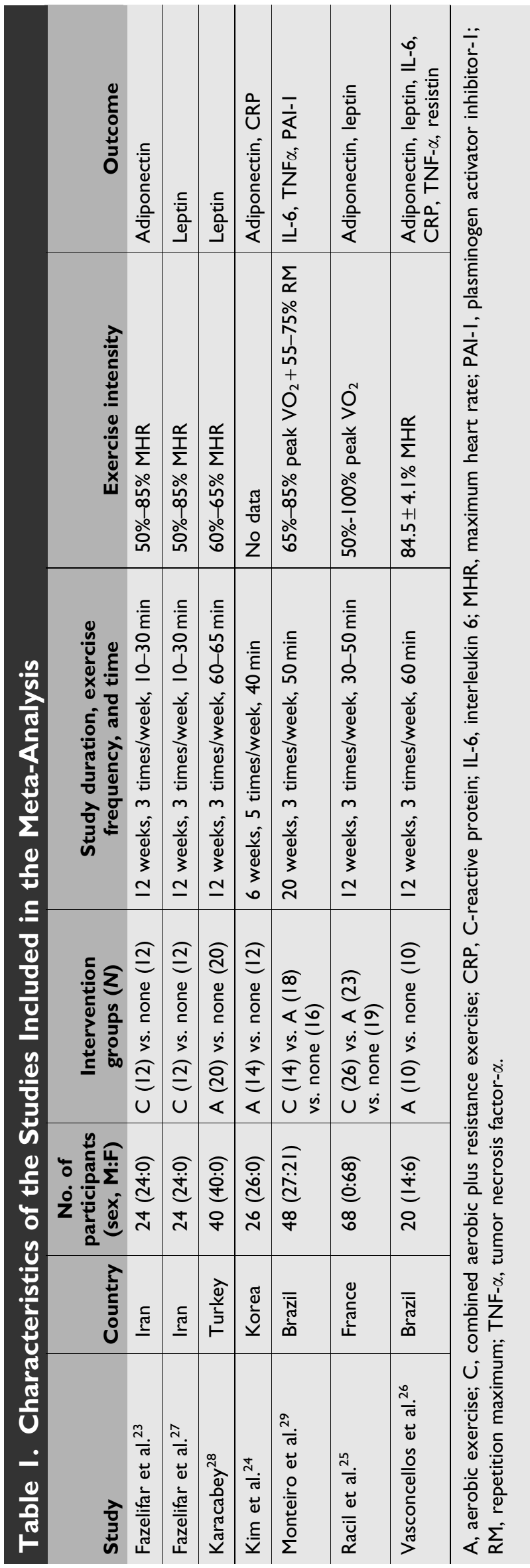

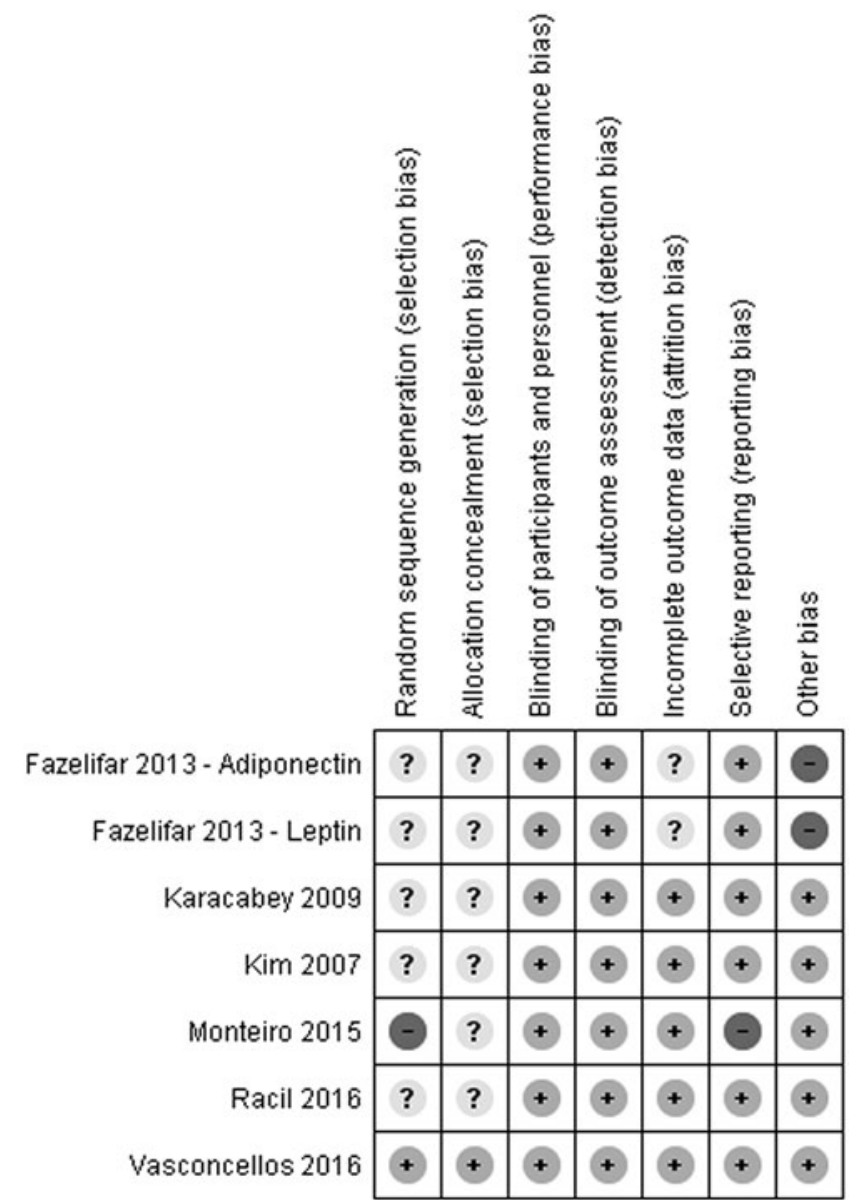

Figure 2. Risk of bias identified in the studies included in the meta-analysis. Low risk of bias is indicated by "+,", high risk of bias "_", unclear risk of bias "?".

intervention group. Two studies comparing TNF- $\alpha$ levels reported discordant results.

The METANINF command was used to assess the impact of each study on the pooled effect on leptin and adiponectin outcome in our meta-analysis. For data regarding leptin, removing one study at a time did not alter the results, indicating a minor effect of individual studies on the pooled estimation and result consistency. Regarding adiponectin, a nonsignificant increase in favor of the intervention group emerged after separately deleting the studies by Kim et al. ${ }^{24}$ and Racil et al. ${ }^{25}$ (Fig. 6 and Table 2).

\section{Discussion}

This study analyzed the effects of exercise on plasma concentration of inflammatory markers in children with obesity. Our findings show that the intervention consisting in physical exercise, without concomitant dietary modification or other lifestyle changes, results in an increase in adiponectin and a decrease in leptin and IL-6 plasma levels. Such changes correspond to a reduction of systemic inflammation associated with obesity.

To the best of our knowledge, there exist two metaanalyses, conducted by García-Hermoso et al., ${ }^{30,31}$ that 


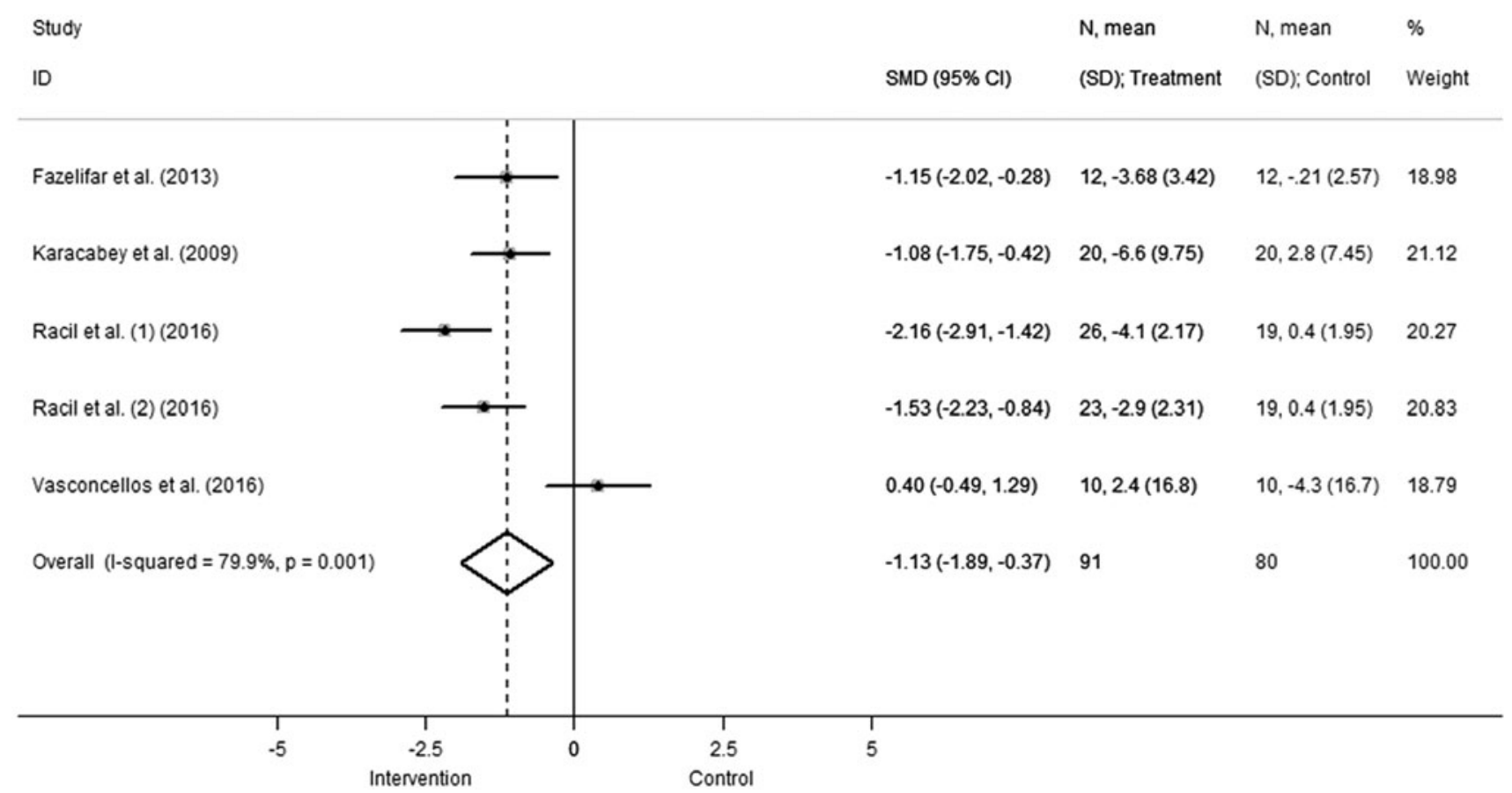

Figure 3. Change in leptin concentration. Squares and the horizontal lines represent the measures of effect, that is, SMD, and associated confidence intervals for each of the studies and the diamond indicates the summary measure. SMD, standardized mean difference.

tested a hypothesis similar to ours; their authors concluded that, in adolescents who had overweight or obesity, exercise increased adiponectin but did not influence significantly leptin or CRP levels. Two striking differences, however, between our meta-analysis and the previously published analyses are evident. First, we applied a more stringent assessment of the quality and risk of bias of included trials. Importantly, it allowed us to identify publications by authors that presumably focused on quantity rather than quality of their articles, as two studies were contemporarily published by the same authors, separately reporting the effects of intervention on leptin and adiponectin levels in the same study

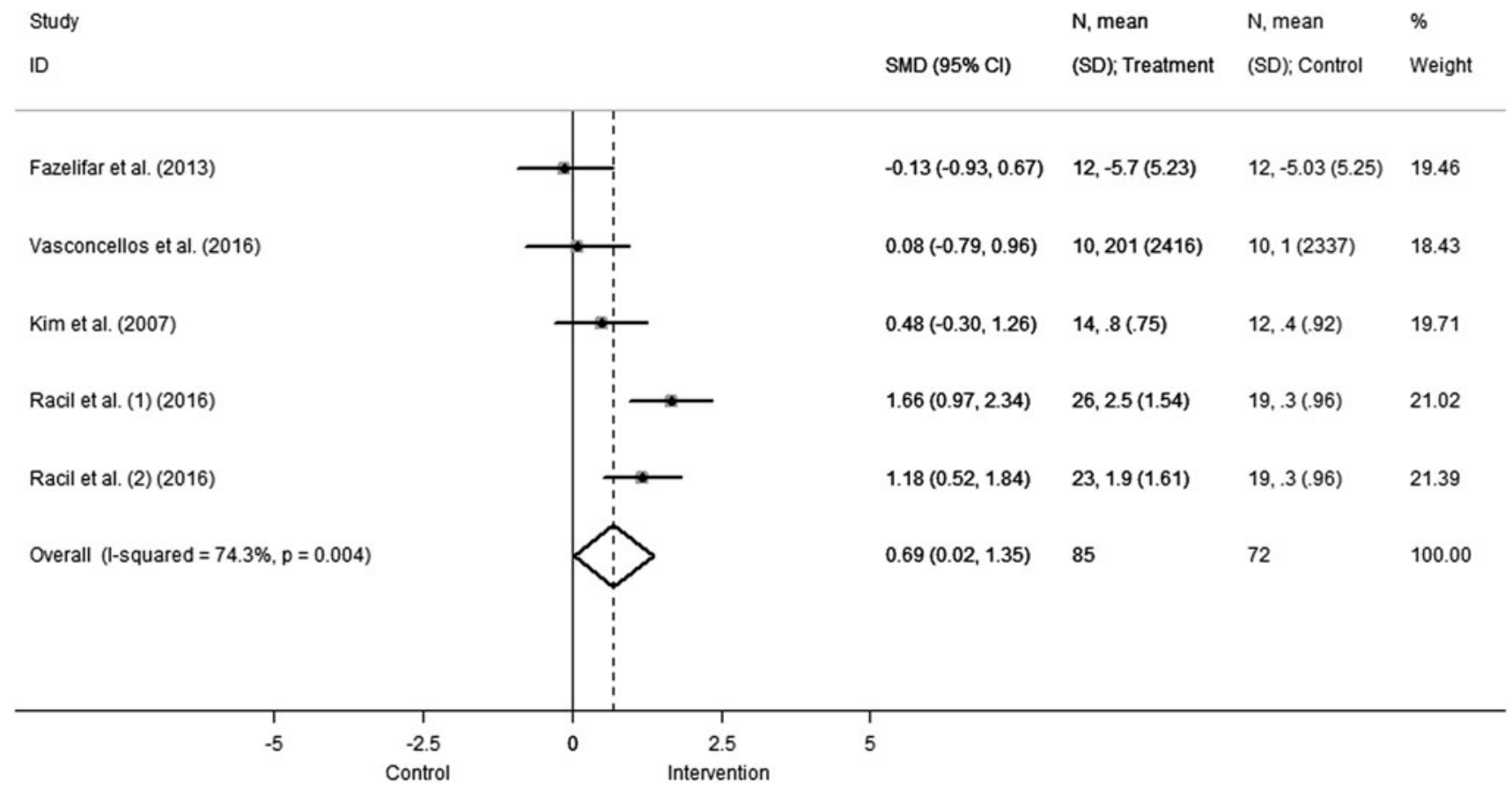

Figure 4. Change in adiponectin concentration. Squares and the horizontal lines represent the measures of effect, that is, SMD, and associated confidence intervals for each of the studies and the diamond indicates the summary measure. 


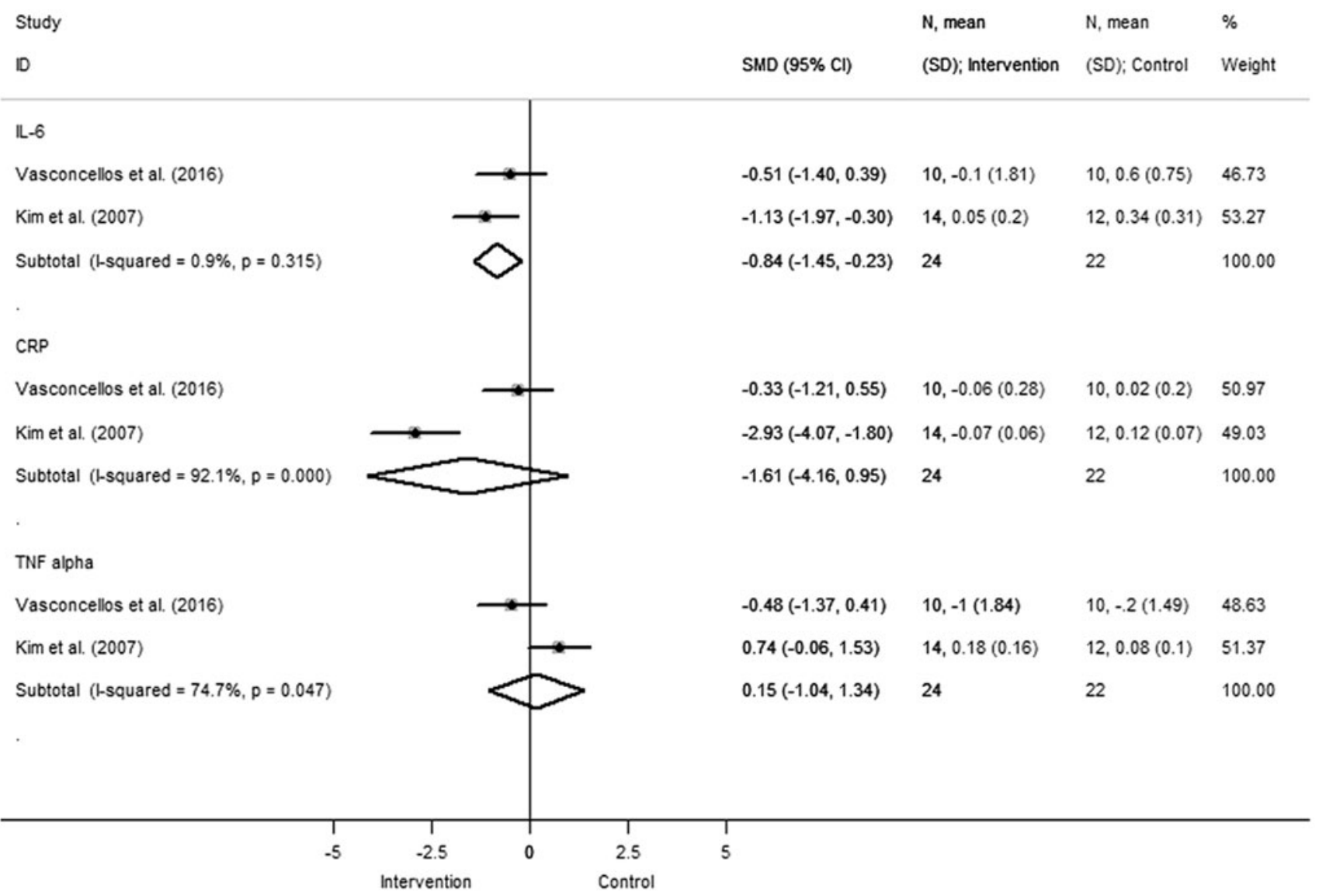

Figure 5. Change in IL-6, CRP, and TNF concentration. Squares and the horizontal lines represent the measures of effect, that is, SMD, and associated confidence intervals for each of the studies and the diamond indicates the summary measure.

group. ${ }^{23,27}$ Segmented publications, indeed, pose a risk to the strength of future recommendations based on meta-analyses. Second, the analyses by García-Hermoso et al. included both children with overweight and children with obesity. There are significant differences between these two groups in circulating levels of adipokines and inflammatory markers in relationship to BMI, suggesting that these biomarkers may change from lean to overweight and from overweight to obesity. ${ }^{32}$ Hence, from a biochemical perspective, these two groups can present different profiles of adipokines and metabolic activity. Such a lack of homogeneity in terms of participants can make it difficult to provide a meaningful
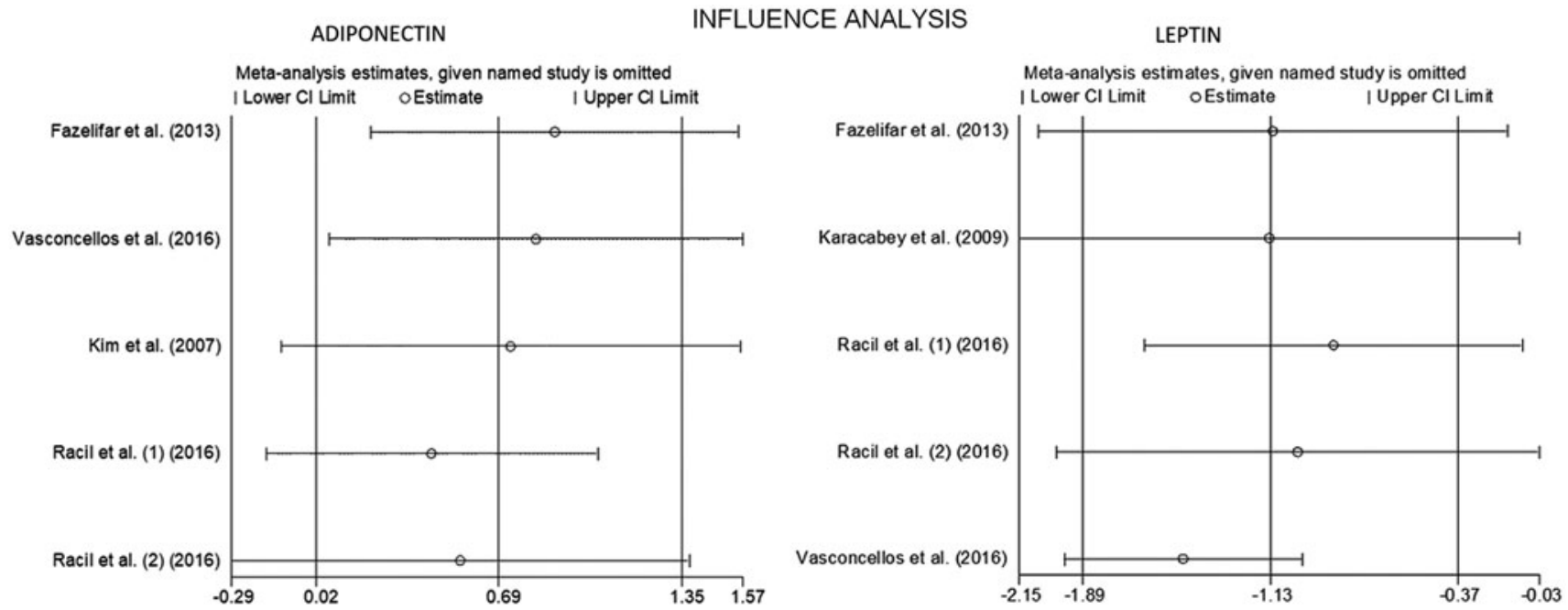

Figure 6. Influence analysis for adiponectin and leptin. 


\begin{tabular}{|c|c|c|c|c|}
\hline Study omitted & Estimate & $\begin{array}{c}\text { Cl } \\
\text { low }\end{array}$ & $\begin{array}{c}\text { Cl } \\
\text { high }\end{array}$ & $\begin{array}{l}\text { Pooled } \\
\text { estimate }\end{array}$ \\
\hline \multicolumn{5}{|l|}{ Leptin } \\
\hline Fazelifar et al. ${ }^{27}$ & -1.12 & -2.07 & -0.16 & Significant \\
\hline Karacabey ${ }^{28}$ & -1.13 & -2.15 & -0.11 & Significant \\
\hline Racil et al..$^{25}(\mathrm{~A})$ & -0.87 & -1.64 & -0.1 & Significant \\
\hline Racil et al. ${ }^{25}$ (C) & -1.02 & -2.0 & -0.03 & Significant \\
\hline $\begin{array}{l}\text { Vasconcellos } \\
\text { et al. }{ }^{26}\end{array}$ & -1.48 & -1.97 & -1.0 & Significant \\
\hline Combined & -1.13 & -1.89 & -0.37 & Significant \\
\hline \multicolumn{5}{|l|}{ Adiponectin } \\
\hline Fazelifar et al. ${ }^{23}$ & 0.89 & 0.22 & 1.56 & Significant \\
\hline $\begin{array}{l}\text { Vasconcellos } \\
\text { et al. }{ }^{26}\end{array}$ & 0.82 & 0.68 & 1.57 & Significant \\
\hline Kim et al. ${ }^{24}$ & 0.73 & -0.11 & 1.56 & Not significant \\
\hline Racil et al..$^{25}(\mathrm{~A})$ & 0.44 & -0.16 & 1.05 & Not significant \\
\hline Racil et al..$^{25}$ (C) & 0.55 & -0.29 & 1.38 & Not significant \\
\hline Combined & 0.69 & 0.02 & 1.35 & Significant \\
\hline
\end{tabular}

The study of Racil et al. ${ }^{25}$ included groups with two different exercise protocols: A, aerobic exercise; C, concomitant aerobic plus resistance exercise.

$\mathrm{Cl}$, confidence interval.

summary of the intervention outcome. For this reason, we decided to include only studies that recruited children with a BMI greater than the 95th percentile for age and sex, hence with obesity, according to the World Health Organization definition.

When the characteristics of the included studies are taken into consideration, several other differences, apart from baseline BMI, may have influenced the outcome: prepuberty or postpuberty age, sex, and type and duration of exercise. Indeed, substantial heterogeneity was detected for the estimated effects of exercise on adiponectin and leptin levels in our meta-analysis. Other studies offer possible clues that indicate some correlation between adipokine levels in plasma and both sex and type of exercise.

In terms of sex, studies suggest that females with obesity show higher plasma leptin compared with males. ${ }^{6}$ Serum leptin concentrations have been reported to be higher in women than man, ${ }^{33}$ and this difference develops with the onset of puberty, possibly accounting for changes in body composition. ${ }^{34}$ Interestingly, apart from the already discussed functions of leptin, it also appears to play a role in pubertal development, as suggested by the observations that obesity during childhood may lead to early signs of puberty in girls and pubertal delay in boys. ${ }^{35}$ Even though a direct relationship between plasma leptin levels and skel- etal muscle metabolism has not been demonstrated, leptin receptors have been identified in the skeletal muscle. ${ }^{36}$ It has been also found that adiponectin levels in prepubertal boys are higher than postpuberty, ${ }^{37}$ while in prepubertal children with obesity, the levels of adiponectin are lower than in those with normal weight. ${ }^{38}$

Incidentally, one study included in our meta-analysis recruited only girls, ${ }^{24}$ other studies recruited only boys, ${ }^{23,24,27,28}$ others still included children of both sexes and the results were pooled. ${ }^{26,29}$ Of note, all the included studies involved adolescents, that is, children between the age of puberty and legal maturity. Further studies are required to elucidate whether the gender and age-related differences in leptin and adiponectin levels might determine differences in skeletal muscle and adipose tissue responses to exercise or influence the risk profile for hypertension and other cardiovascular morbidities associated with obesity in men and women.

Regarding duration of exercise, the available studies reported disparate findings on the responses of adiponectin and leptin to short- and long-term exercise in sedentary and trained subjects (reviewed by Bouassida et al.). ${ }^{39}$ In addition, the type of activity (aerobic, resistance, or concurrent exercise) can greatly determine the effects of the exercise on body mass and composition. While aerobic exercise is considered optimal for reducing body fat, resistance exercise leads to an increase in lean body mass. ${ }^{40}$ Arguably, both these body composition modifications can prevent chronic diseases related to obesity. Interestingly, data from a recent clinical trial comparing aerobic, resistance, and mixed training effects on health suggested that aerobic training alone was the most efficient mode of exercise for improving metabolic syndrome. ${ }^{41}$ Among the studies included in our meta-analysis, five included aerobic ${ }^{24-26,28,29}$ and four included concurrent, aerobic and resistance exercise groups. ${ }^{23,25,27,29}$ Two studies directly compared aerobic and concurrent exercise, ${ }^{25,29}$ reporting similar decreases in body fat in both training groups with respect to the controls. Only concurrent training induced a significant increase in lean body mass; in the same group, a significantly greater decrease in the plasma leptin/adiponectin ratio was evident. ${ }^{25}$ At this point, the available data are insufficient to draw conclusions regarding the adipokine response to different modes of exercise.

With regard to IL-6, adipocytes and stromal vascular cells in the adipose tissue (including pericytes, supra-adventitial adipose stromal cells, and endothelial cells) ${ }^{42}$ are capable of synthesis and secretion of this cytokine. ${ }^{43}$ Remarkably, IL-6 is released from skeletal muscle, but not from the subcutaneous adipose tissue, during exercise in relationship to exercise intensity. ${ }^{44}$ When these data are taken into consideration, the findings of the studies of interventions consisting in physical exercise, with a modification of body mass and composition, can be difficult to interpret. Even if available data regarding IL-6 level in children with obesity and the consequences of exercise intervention on this inflammatory marker are unclear, data pooled from the 
studies included in the present meta-analysis indicated that there was a significant reduction in IL-6 concentration following exercise in children with obesity.

In children, achieving compliance with recommendations can be difficult (indeed, many participants dropped out of clinical trials included in our meta-analysis). However, this group should be targeted in programs aimed at cardiovascular disease prevention, because of the known association between obesity in childhood and adulthood. ${ }^{1}$ Dieting alone often fails to achieve long-term benefits in individuals with obesity ${ }^{45}$; the possible reason being that weight loss leads to a reduction in leptin levels, leading in turn to an increase in hunger. ${ }^{46}$ The role of physical activity and exercise in health and disease extends well beyond the effects on body weight. Indeed, both weight management and increased physical activity are currently recommended for cardiovascular disease prevention. ${ }^{47}$ If adipose tissue is regarded as an endocrine organ responding to changes in the metabolic status, then the systemic regulation of its activity and modification of the target organ response, rather than mere reduction in the amount of body fat, should allow for reduction in obesity-related inflammation for chronic disease prevention. In light of discussed data, physical exercise emerges as an optimal measure for achieving this goal.

At present, the evaluation of interventions aimed at eliminating obesity for cardiovascular and metabolic disease prevention is commonly based on changes in anthropometric parameters, body composition, or lipid and glucose levels. These outcome measures may be insufficient. As a matter of fact, insulin sensitivity, lipid, inflammation, and the hormonal profile can present normal cardioprotective traits in as much as $30 \%$ of individuals with obesity. Interestingly, individuals with such a metabolically healthy obese phenotype have higher physical activity and fitness levels when compared with individuals with obesity who present with the metabolic syndrome. ${ }^{48}$ Two recent systematic reviews and meta-analyses indicated an increased risk of cardiovascular disease and death in patients with obesity even in the absence of metabolic abnormalities. ${ }^{49,50}$ However, when data were adjusted to account for physical level, individuals with obesity who were fit did not have an increased risk of mortality compared with the normal weight fit group. ${ }^{51}$ Importantly, the increase in circulating leptin observed in obesity is associated with leptin resistance developing at both central nervous system and adipose tissue level. However, in the light of the recent conclusion that direct activation of adipocyte leptin receptors has little effect on cell metabolism in vivo, ${ }^{52}$ the indirect influence of leptin on adipocytes can play a greater role in adipocyte activity control. Indeed, there is evidence for leptin inhibiting insulin secretion and impairing adipocyte insulin responsiveness in vitro. ${ }^{53}$ This influence was corroborated by the results of the clinical study, in which reduced leptin plasma levels following moderate weight loss in obese adults correlated with significant improvement of insulin resistance. ${ }^{54}$ Taken together, these observations again un- derscore the role of physical activity for the prevention of chronic metabolic and cardiovascular disease.

If individuals with obesity can be metabolically healthy, then individuals with normal weight can have metabolic obesity. Despite their normal weight, patients with an accumulation of visceral fat are at increased risk for type 2 diabetes. ${ }^{55}$ In light of these findings, several markers, such as waist circumference or visceral adiposity index, emerged as potentially useful tools for classifying and stratifying cardiovascular risk based on body composition rather than body mass. ${ }^{56}$ In adolescents, the levels of adiponectin and other inflammatory markers correlated with adiposity evaluated by waist circumference and subscapular and triceps skinfold measurements. ${ }^{57}$ Regarding this connection, it would be important for the future studies to use parameters describing adiposity rather than weight status for patient inclusion and randomization when evaluating the effects of interventions on levels of adipokines in plasma.

\section{Limitations of the Study}

The results of the present meta-analysis should be considered with caution, as several limitations can be identified. First and foremost, the character of intervention, consisting in supervised physical exercise, does not allow for blinding of participants and personnel. However, even if it was not explicitly specified in the included studies, we assumed that the laboratory personnel involved in the outcome assessment, that is, blood sample analysis, was blinded to patients' allocation; hence, we identified a low risk of performance bias. Second, all the included studies had a small sample size (less than 100 participants). As discussed above, a high level of heterogeneity, regarding sex, type, intensity, and duration of exercise, does not allow for generalization, and the small numbers of studies and participants make the adjustment of data infeasible. Third, while STATA software allows to test for publication bias, the widely accepted funnel plot method has several limitations, as true heterogeneity, poor methodological design or chance can account for visual funnel plot asymmetry. According to Higgins et al., ${ }^{20}$ the tests for funnel plot asymmetry should be used only when there are at least 10 studies included in the metaanalysis, otherwise the power of the tests is too low to distinguish chance from real asymmetry.

Another major hurdle in the study of adipokines in childhood obesity and analysis of the response to exercise is that leptin and adiponectin expression differs in relationship to sex and age; in particular, hormonal differences between boys and girls and between prepubertal and postpubertal age in both sexes are major determinants of plasma adipokines. None of the studies included in the present meta-analysis specifically aimed to address these issues while investigating or interpreting the effects of physical exercise on adiponectin and leptin levels. Finally, even though exercise positively influenced the levels of adiponectin and leptin in plasma, the significance of the adipokine level modification as the outcome measure of 
the interventions aimed at weight and cardiovascular risk reduction remains unknown.

\section{Conclusion}

Physical exercise, without concomitant dietary modification or other lifestyle changes, is associated with an increase in adiponectin and a decrease in leptin and IL-6 plasma levels in children with obesity, thus reducing the obesity-associated systemic inflammation. Considering the role that adipose tissue-derived cytokines play in metabolic homeostasis, it is tempting to suggest that these and possibly other inflammatory factors may be related to metabolic syndrome and cardiovascular disease. Hence, the usefulness of evaluating adipokines in plasma should continue to be explored in clinical studies both in children and adults. Studies with a uniform intervention protocol and outcome measurements are required to put our new knowledge on adipose tissue biology into a clinical perspective.

\section{Author Disclosure Statement}

No competing financial interests exist.

\section{References}

1. Juonala M, Magnussen CG, Berenson GS, et al. Childhood adiposity, adult adiposity, and cardiovascular risk factors. $N$ Engl $J$ Med 2011;365:1876-1885.

2. Lombardo FL, Spinelli A, Lazzeri G, et al; OKkio alla SALUTE Group 2010. Severe obesity prevalence in 8- to 9-year-old italian children: A large population-based study. Eur J Clin Nutr 2015;69: 603-608.

3. Kershaw EE, Flier JS. Adipose tissue as an endocrine organ. $J$ Clin Endocrinol Metab 2004;89:2548-2556.

4. Deng Y, Schere PE. Adipokines as novel biomarkers and regulators of metabolic syndrome. Ann N Y Acad Sci 2010;1212:E1-E19.

5. Jaleel A, Aheed B, Jaleel S, et al. Association of adipokines with obesity in children and adolescents. Biomark Med 2013;7:731-735.

6. Aycan Z, Berberoğlu M, Ocal G, et al. Relationship between plasma leptin, insulin and tumor necrosis factor alpha in obese children. J Pediatr Endocrinol Metab 2005;18:275-284.

7. Lee JH, Reed DR, Price RA. Leptin resistance is associated with extreme obesity and aggregates in families. Int J Obes Relat Metab Disord 2001;25:1471-1473.

8. Shehzad A, Iqbal W, Shehzad O, Lee YS. Adiponectin: Regulation of its production and its role in human diseases. Hormones 2012; 11:8-20.

9. Kwon H, Pessin JE. Adipokines mediate inflammation and insulin resistance. Front Endocrinol (Lausanne) 2013;4:71.

10. Schoppen S, Riestra P, García-Anguita A, et al. Leptin and adiponectin levels in pubertal children: Relationship with anthropometric variables and body composition. Clin Chem Lab Med 2010; 48:707-711.

11. Maachi M, Piéroni L, Bruckert E, et al. Systemic low-grade inflammation is related to both circulating and adipose tissue TNFalpha, leptin and IL-6 levels in obese women. Int J Obes Relat Metab Disord 2004;28:993-997.
12. Bastard JP, Maachi M, Lagathu C, et al. Recent advances in the relationship between obesity, inflammation, and insulin resistance. Eur Cytokine Netw 2006;17:4-12.

13. Han TS, Lean ME. A clinical perspective of obesity, metabolic syndrome and cardiovascular disease. JRSM Cardiovasc Dis 2016; 5:2048004016633371.

14. Allison DB, Zannolli R, Faith MS, et al. Weight loss increases and fat loss decreases all-cause mortality rate: Results from two independent cohort studies. Int J Obes Relat Metab Disord 1999;23: $603-611$.

15. Harrington M, Gibson S, Cottrell RC. A review and meta-analysis of the effect of weight loss on all-cause mortality risk. Nutr Res Rev 2009;22:93-108.

16. Baillot A, Romain AJ, Boisvert-Vigneault K, et al. Effects of lifestyle interventions that include a physical activity component in class II and III obese individuals: A systematic review and metaanalysis. PLoS One 2015;10:e0119017.

17. Woodcock J, Franco OH, Orsini N, Roberts I. Non-vigorous physical activity and all-cause mortality: Systematic review and meta-analysis of cohort studies. Int J Epidemiol 2011;40:121-138.

18. Pedersen BK. Body mass index-independent effect of fitness and physical activity for all-cause mortality. Scand J Med Sci Sports 2007;17:196-204.

19. Moher D, Liberati A, Tetzlaff J, et al. Preferred reporting items for systematic reviews and meta-analyses: The PRISMA statement. BMJ 2009;339:b2535.

20. Higgins JPT, Altman DG, Sterne JAC, editors. Assessing risk of bias in included studies. In: Higgins JPT, Green S, editors. Cochrane Handbook for Systematic Reviews of Interventions Version 5.1.0 [Internet]. The Cochrane Collaboration; 2011. Available at: www.handbook.cochrane.org (last accessed September 10, 2017).

21. Review Manager (RevMan) [Computer program]. Version 5.3. Copenhagen: The Nordic Cochrane Centre, The Cochrane Collaboration, 2014.

22. DerSimonian R, Laird N. Meta-analysis in clinical trials. Control Clin Trials 1986;7:177-188.

23. Fazelifar S, Ebrahim K, Sarkisian V. Effect of concurrent training and detraining on anti-inflammatory biomarker and physical fitness levels in obese children. Rev Bras Med Esporte [Internet] 2013;19: 349-54. Available at: www.scielo.br/scielo.php?script=sci_arttext \&pid=S1517-86922013000500010\&lng=en (last accessed September 10, 2017).

24. Kim ES, Im JA, Kim KC, et al. Improved insulin sensitivity and adiponectin level after exercise training in obese Korean youth. Obesity (Silver Spring) 2007;15:3023-3030.

25. Racil G, Zouhal H, Elmontassar W, et al. Plyometric exercise combined with high-intensity interval training improves metabolic abnormalities in young obese females more so than interval training alone. Appl Physiol Nutr Metab 2016;41(1):103-109.

26. Vasconcellos F, Seabra A, Cunha F, et al. Health markers in obese adolescents improved by a 12 -week recreational soccer program: A randomised controlled trial. J Sports Sci 2016;34:564-575.

27. Fazelifar S, Ebrahim K, Sarkisian V. Effect of exercise training and detraining on leptin levels in obese young boys. Med Sport 2013;66:325-337.

28. Karacabey K. The effect of exercise on leptin, insulin, cortisol and lipid profiles in obese children. $J$ Int Med Res 2009;37:14721478.

29. Monteiro PA, Chen KY, Lira FS, et al. Concurrent and aerobic exercise training promote similar benefits in body composition and metabolic profiles in obese adolescents. Lipids Health Dis 2015; $14: 153$. 
30. García-Hermoso A, Ceballos-Ceballos RJM, Poblete-Aro CE, et al. Exercise, adipokines and pediatric obesity: A meta-analyis of randomized controlled trials. Int J Obes (Lond) 2017;41:475482.

31. García-Hermoso A, Sánchez-López M, Escalante Y, et al. Exercise-based interventions and C-reactive protein in overweight and obese youths: A meta-analysis of randomized controlled trials. Pediatr Res 2016;79:522-527.

32. Mantovani RM, Rocha NP, Magalhães DM, et al. Early changes in adipokines from overweight to obesity in children and adolescents. $J$ Pediatr (Rio J) 2016;92:624-630.

33. Garaulet M, Perex-Llamas F, Fuente T, et al. Anthropometric, computed tomography and fat cell data in an obese population: Relationship with insulin, leptin, tumor necrosis factor-alpha, sex hormone-binding globulin and sex hormones. Eur $J$ Endocrinol 2000;143:657-666.

34. Ahmed ML, Ong KK, Morrell DJ, et al. Longitudinal study of leptin concentrations during puberty: Sex differences and relationship to changes in body composition. J Clin Endocrinol Metab 1999;84:899-905.

35. Burt Solorzano CM, McCartney CR. Obesity and the pubertal transition in girls and boys. Reproduction 2010;140:399-410.

36. Guerra B, Santana A, Fuentes T, et al. Leptin receptors in human skeletal muscle. J Appl Physiol 2007;102:1786-1792.

37. Bottner A, Kratzsch J, Muller G, et al. Gender differences of adiponectin levels develop during the progression of puberty and are related to serum androgen levels. J Clin Endocrinol Metab 2004;89:4053-4061.

38. Paltoglou G, Schoina M, Valsamakis G, et al. Interrelations among the adipocytokines leptin and adiponectin, oxidative stress and aseptic inflammation markers in pre- and early-pubertal normalweight and obese boys. Endocrine 2017;55:925-933.

39. Bouassida A, Chamari K, Zaouali M, et al. Review on leptin and adiponectin responses and adaptations to acute and chronic exercise. Br J Sports Med 2010;44:620-630.

40. Willis LH, Slentz CA, Bateman LA, et al. Effects of aerobic and/or resistance training on body mass and fat mass in overweight or obese adults. J Appl Physiol 2012;113:1831-1837.

41. Bateman LA, Slentz CA, Willis LH, et al. Comparison of aerobic versus resistance exercise training effects on metabolic syndrome (from the Studies of a Targeted Risk Reduction Intervention Through Defined Exercise - STRRIDE-AT/RT). Am J Cardiol 2011;108:838-844.

42. Zimmerlin L, Donnenberg VS, Rubin JP, et al. Mesenchymal markers on human adipose stem/progenitor cells. Cytometry 2013; 83A:134-140.

43. Eder K, Baffy N, Falus A, et al. The major inflammatory mediator interleukin-6 and obesity. Inflamm Res 2009;58:727-736.

44. Petersen AM, Pedersen BK. The anti-inflammatory effect of exercise. J Appl Physiol 2005;98:1154-1162.

45. Flynn MA, McNeil DA, Maloff B, et al. Reducing obesity and related chronic disease risk in children and youth: A synthesis of evidence with "best practice" recommendations. Obes Rev 2006;7 (Suppl 1):7-66.
46. Myers MG Jr, Leibel RL, Seeley RJ, Schwartz MW. Obesity and leptin resistance: Distinguishing cause from effect. Trends Endocrinol Metab 2010;21:643-651.

47. National Cholesterol Education Program (NCEP) Expert Panel on Detection, Evaluation, and Treatment of High Blood Cholesterol in Adults (Adult Treatment Panel III). Third Report of the National Cholesterol Education Program (NCEP) Expert Panel on Detection, Evaluation, and Treatment of High Blood Cholesterol in Adults (Adult Treatment Panel III) final report. Circulation 2002; 106:3143-3421.

48. Faroog A, Sorathia S, Shaharyar S, et al. Metabolically healthy obesity and the Fit/Fat phenotype: Associations with mortality, subclinical cardiovascular disease and approach to treatment. J Metabolic Synd 2015;4:2.

49. Roberson LL, Aneni EC, Maziak W. Beyond BMI: The "Metabolically healthy obese" phenotype \& its association with clinical/ subclinical cardiovascular disease and all-cause mortality-A systematic review. BMC Public Health 2014;14:14.

50. Kramer CK, Zinman B, Retnakaran R. Are metabolically healthy overweight and obesity benign conditions?: A systematic review and meta-analysis. Ann Intern Med 2013;159:758-769.

51. Barry V, Baruth M, Beets MW, et al. Fitness vs. fatness on allcause mortality: A meta-analysis. Prog Cardiovasc Dis 2014;56: 382-390.

52. Harris RB. Direct and indirect effects of leptin on adipocyte metabolism. Biochim Biophys Acta 2014;1842:414-423.

53. Muller G, Ertl J, Gerl M, et al. Leptin impairs metabolic actions of insulin in isolated rat adipocytes. J Biol Chem 1997;272: 10585-10593.

54. Wang TN, Chang WT, Chiu YW, et al. Relationships between changes in leptin and insulin resistance levels in obese individuals following weight loss. Kaohsiung J Med Sci 2013;29:436-443.

55. Succurro E, Marini MA, Frontoni S, et al. Insulin secretion in metabolically obese, but normal weight, and in metabolically healthy but obese individuals. Obesity (Silver Spring) 2008;16:1881-1886.

56. Amato MC, Guarnotta V, Giordano C. Body composition assessment for the definition of cardiometabolic risk. J Endocrinol Invest 2013;36:537-543.

57. Rubin DA, McMurray RG, Harrell JS, et al. Do surrogate markers for adiposity relate to cytokines in adolescents? J Investig Med 2008;56:786-792.

Address correspondence to: Felice Sirico, MD Department of Public Health, Human Anatomy, and Sports Medicine Section University of Naples "Federico II" via S. Pansini 5, Building 20 Naples 80131 Italy

E-mail: sirico.felice@gmail.com 